\title{
Pólipos vesiculares: correlación entre hallazgos ecográficos e histopatológicos
}

\author{
Alex Escalona $\mathbf{P}^{1}$, Francisca León $\mathbf{G}^{1}$, Felipe Bellolio $\mathbf{R}^{1}$, \\ Fernando Pimentel $M^{1}$, Matías $G$ uajardo $B^{1}$, Rubén \\ $\mathrm{Gennero}^{1}$, Juan Pablo Cruz $\mathrm{Q}^{1}$, Paola Viviani G ${ }^{2, a}$, \\ Luis Ibáñez $A^{1}$.
}

\section{Gallbladder polyps: correlation between ultrasonographic and histopathological findings}

Background: Gallbladder polyps are becoming a common finding. The management of these polyps is complicated considering that they can bear malignant lesions. Aim: To analyze the ultrasonographic and histopathologic findings of patients operated due to gallbladder polyps. Patients and methods: The records of patients with ultrasonographic diagnosis of gallbladder polyp and that underwent cholecystectomy in a thirteen years period were reviewed, collecting their demographic, ultrasonographic and histopathological data. Results: One hundred and twenty three patients were operated. The mean age was $44 \pm 13$ years, and $69 \%$ were women. The mean size of polyps in ultrasonography was $7.3 \pm 5 \mathrm{~mm}$. Histopathology confirmed the presence of polyps in $79 \%$ of patients, with a mean size and number of lesions of $5.1 \pm 3.8 \mathrm{~mm}$ and $2.1 \pm 2$, respectively. Nine percent of polyps were greater than $10 \mathrm{~mm}$, and single polyps were significantly larger than the multiple ones ( $p$ $=0.003$ ). Four cases of adenoma (3.2\%) were diagnosed; one of them had in situ carcinoma. All were single and larger than $10 \mathrm{~mm}$. We found a significant correlation between ultrasonographic and histopathological polyp size determination ( $r=0.47 ; p=0.002$ ). Polyp size was also a predictor of the presence of adenoma ( $p=0.043$; confidence intervals: 1.006-1.424). Conclusions: There is a good correlation between the size of the gallbladder polyp in ultrasonography and the size in the histopathology report. Gallbladder adenoma is uncommon and it correlates with the size of the polyp. In this series, size was the only predictor of the presence of adenoma (Rev Méd Chile 2006; 134: 1237-42).

(Key words: Adenomatous polyps; Gallbladder neoplasms; Polyps)

Recibido el 7 de diciembre, 2005. Aceptado el 4 de abril, 2006.

${ }^{1}$ Departamento de Cirugía Digestiva, División de Cirugía. ${ }^{2}$ Departamento de Salud Pública, Facultad de Medicina, Pontificia Universidad Católica de Chile. Santiago, Chile.

aBioestadística

Correspondencia a: Dr. Alex Escalona P. Marcoleta 367,

Santiago. Fono: 56-2-3543462. Fax: 56-2-6382793.

E-mail: aescalon@puc.cl 
$\mathrm{E}^{1}$ pólipo vesicular constituye un diagnóstico cada vez más frecuente, principalmente como hallazgo en una ecotomografía abdominal pedida por otra causa ${ }^{1-4}$. Corresponde a cualquier elevación de la mucosa de la vesícula biliar. Se clasifican en lesiones malignas (adenocarcinoma, metástasis y otras) y benignas, siendo éstas últimas tumorales (adenoma, leiomioma, lipoma) o pseudotumorales (pólipos de colesterol, adenomiomas, pólipos inflamatorios, pólipos hiperplásticos, otros) ${ }^{5}$.

Estudios epidemiológicos reportan una prevalencia ecográfica de lesiones polipoideas entre $4,0 \%$ y $5,6 \% 2,6$. Por otra parte, su incidencia en colecistectomías alcanza hasta 13,8\% en diferentes series internacionales ${ }^{7,8}$. En Chile la incidencia corresponde a 0,6\%-1\%9,10.

La importancia de un diagnóstico preciso tiene relación con la posibilidad de desarrollar una neoplasia maligna a partir de un adenoma ${ }^{11-13}$. Además, es sabido que los adenocarcinomas vesiculares podrían también adoptar formas polipoideas en la ecotomografía. Esta patología constituye un problema de salud pública importante en nuestro país, con una prevalencia descrita en colecistectomías de 3,4\% en mujeres y 1,32\% en hombres ${ }^{14}$.

Los objetivos de este estudio son correlacionar los hallazgos ecográficos e histopatológicos en pacientes operados por pólipo vesicular e identificar factores predictores de adenoma vesicular.

\section{PACIENTES Y MÉTODO}

Se revisaron, retrospectivamente, las fichas de los pacientes sometidos a colecistectomía por pólipo vesicular, desde mayo de 1991 a julio de 2004, en el Hospital Clínico de la Pontificia Universidad Católica de Chile. Se registraron los datos demográficos de cada paciente y los informes de la ecotomografía abdominal. Se consideró pólipo a toda lesión solevantada hacia el lumen vesicular con ecogenicidad similar a su pared, con o sin pedículo, no desplazable y sin sombra acústica. Se revisaron los informes histopatológicos y se correlacionaron con la información ecográfica.

Los datos fueron ingresados y analizados en base de datos en el programa SPSS 10.0 para Windows. Las variables continuas están expresa- das como promedio y desviación estándar, y se analizaron mediante análisis de varianza (ANOVA) $\mathrm{y}$ test $\mathrm{t}$ de Student. Para tablas de contingencia se usaron test de Chi-cuadrado o test exacto de Fisher. Se evaluó la relación entre variables continuas mediante el coeficiente de correlación de Pearson. Para la identificación de factores predictores de adenoma en pólipos vesiculares se usó análisis de regresión logística. Se consideró estadísticamente significativo un valor $\mathrm{p}<0,05$.

\section{RESUlTADos}

En el periodo estudiado, 123 pacientes fueron sometidos a colecistectomía por pólipo vesicular, 85 de ellos (69\%) de sexo femenino. Todos los pacientes fueron operados por vía laparoscópica. No hubo conversión a cirugía abierta ni morbilidad quirúrgica. La edad promedio fue $44 \pm 13$ años. De acuerdo a la ecotomografía abdominal, el número de pólipos fue $1,3 \pm 0,7$ por paciente, y el tamaño promedio fue $7,3 \pm 5 \mathrm{~mm}$.

El estudio histopatológico demostró la presencia de pólipos en 94 de los 123 pacientes (76,4\%), mientras que en los 29 restantes $(23,6 \%)$ no se detectaron pólipos en la pieza operatoria. De los 94 pacientes con pólipos, se diagnosticaron adenomas en cuatro casos (4,3\%), uno de los cuales demostró focos de carcinoma in situ. El resto correspondió a pseudotumores compuestos por pólipos de colesterol, adenomiosis y colesterolosis (Tabla 1). En todos los pacientes con adenoma vesicular la lesión ecográfica e histopatológica fue

\section{Tabla 1. D escripción de pólipos vesiculares en la histopatología ( $n=94)$}

\begin{tabular}{|lrr|}
\hline Tipo de pólipo & $\mathrm{n}$ & $\%$ \\
\hline Pseudotumor & & \\
$\quad$ Pólipos de colesterol & 66 & 70,2 \\
Adenomioma & 2 & 2,1 \\
Colesterolosis & 22 & 23,4 \\
Tumor & & \\
Adenoma & $4^{*}$ & 4,3 \\
\hline
\end{tabular}

*Incluye a un paciente con adenoma vesicular con focos de carcinoma in situ. 
Tabla 2. Análisis de edad según número, tamaño y tipo de pólipos en la histopatología ( $n=94$ )

\begin{tabular}{|lcccc|}
\hline & Categoría & $\begin{array}{c}\mathrm{n} / \% \text { (del total } \\
\text { de pólipos) }\end{array}$ & $\begin{array}{c}\text { Edad en años } \\
\text { (promedio } \pm \mathrm{ds} \text { ) }\end{array}$ & Valor p \\
\hline № de pólipos & 1 & $50 / 52,9$ & $44 \pm 14$ & NS \\
& $\geq 2$ & $44 / 47,1$ & $45 \pm 13$ & NS \\
Tamaño (mm) & $\leq 5$ & $67 / 71$ & $43 \pm 13$ & \\
& $6-10$ & $19 / 20,3$ & $44 \pm 12$ & NS \\
Tipo de tumor & $>10$ & $8 / 8,7$ & $47 \pm 17$ & \\
& Pseudotumor & $90 / 95,7$ & $43 \pm 13$ & \\
\hline
\end{tabular}

NS: No significativo.

única y mayor de $10 \mathrm{~mm}$. En ningún paciente se demostró la presencia de carcinoma invasor.

Se constató pólipos múltiples en $47,1 \%$ de los casos. El tamaño y número de las lesiones por paciente fue en promedio $5,1 \pm 3,8 \mathrm{~mm}$ y $2,1 \pm 2$ pólipos, respectivamente. El 8,7\% de los pólipos fueron mayores de $10 \mathrm{~mm}$. No se demostraron diferencias significativas para la edad según el tipo ( $\mathrm{p}=0,62)$, tamaño $(\mathrm{p}=0,79)$ y número de pólipos ( $\mathrm{p}=0,83$ ) (Tabla 2). Tampoco se demostró diferencia estadísticamente significativa de sexo según el tipo de pólipo $(p=0,76)$. El tamaño promedio de las lesiones únicas fue significativamente mayor que el de las múltiples $(p=0,003)$ (Tabla 3), sin encontrar asociación significativa entre el tipo de pólipo y el número ( $p=0,237$ ).
El coeficiente de correlación ( $\mathrm{r}$ ) entre tamaño del pólipo por ecotomografía y el tamaño en el estudio histopatológico es 0,47 ( $p=0,002$ ), considerado como aceptable (Figura 1).

Tabla 3. Análisis del tamaño y número de pólipos vesiculares en la histopatología $(n=94)$

\begin{tabular}{|cc|}
\hline № de pólipos & $\begin{array}{c}\text { Tamaño en } \mathrm{mm} \\
\text { (promedio } \pm \mathrm{ds} \text { ) }\end{array}$ \\
\hline 1 & $6,7 \pm 0,9$ \\
$\geq 2$ & $3,5 \pm 0,4$ \\
\hline
\end{tabular}

$(p=0,003)$

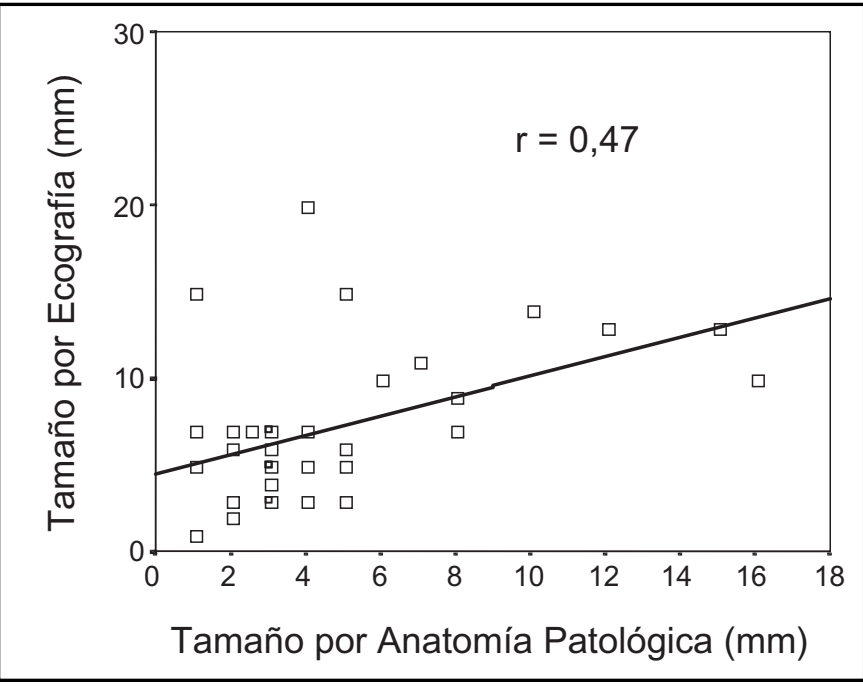

Figura 1. Correlación entre el tamaño ecográfico y el tamaño histopatológico del pólipo vesicular. 
Tabla 4. Análisis de regresión logística para predicción de adenoma

\begin{tabular}{|lcc|}
\hline Variables & OR & IC $(95,0 \%)$ \\
\hline Edad (años) & 1,005 & $0,931-1,085$ \\
Tamaño de pólipo (mm) & 1,197 & $1,006-1,424$ \\
Sexo (femenino) & 0,489 & $0,045-5,263$ \\
\hline
\end{tabular}

Se diagnosticó colelitiasis (no descrita en la ecotomografía preoperatoria) en $14 \%$ de los casos; no se demostró asociación entre su presencia y el tipo de tumor ( $\mathrm{p}=0,052)$.

De acuerdo al análisis de regresión logística el tamaño de los pólipos fue identificado como el único factor predictor de adenoma en el estudio histopatológico, con un OR de 1,197 (IC 95\% =1,006-1,424) (Tabla 4).

\section{Discusión}

El hallazgo de un pólipo vesicular implica una toma de decisiones controversial, debido a la posibilidad de ser una forma de presentación de un adenocarcinoma o de desarrollar una neoplasia maligna a partir de él. El diagnóstico ecográfico plantea un desafío importante al respecto, debido a que frecuentemente se detecta en pacientes que son sometidos a ese examen por otras razones ${ }^{1-4}$.

Se ha descrito que la sensibilidad de la ecotomografía para la detección de pólipos vesiculares fluctúa entre $32 \%$ y $90 \% 3,8$, con una especificidad de $93,9 \%^{8}$. Si bien, este método tendría ventajas sobre otros para detectar lesiones pequeñas, hay que considerar que el rendimiento de la ecotomografía igualmente depende del tamaño de las lesiones, de la presencia de colelitiasis, de la experiencia del operador y de la calidad del instrumento ${ }^{14}$. En esta serie, todas las lesiones mayores de $10 \mathrm{~mm}$ fueron informadas en la ecografía como pólipos de al menos ese tamaño. Además, se pudo establecer que la ecotomografía abdominal aportaría información segura respecto del tamaño lesional y que el tamaño también orientaría a la naturaleza tumoral del pólipo. Boulton et $\mathrm{al}^{4}$ mencionan característi- cas ultrasonográficas que permitirían diferenciar pólipos de colesterol de otras lesiones benignas, los que característicamente son pequeños, múltiples e hiperecoicos a la ecotomografía por su contenido lipídico. Para confirmarlo, se necesitan estudios prospectivos.

En esta serie, todas las lesiones adenomatosas fueron mayores de $10 \mathrm{~mm}$, lo que se confirma con el análisis de regresión logística, que muestra al tamaño como el único predictor de adenoma. Se ha descrito que el tamaño y número de pólipos tienen relación con las características histopatológicas de los pólipos vesiculares y que el tamaño sería el más importante predictor de malignidad en los pólipos vesiculares ${ }^{15}$, con hasta $88 \%$ de las lesiones neoplásicas con tamaño mayor a 10 $\mathrm{mm}^{1,6}$. Por otra parte, estudios plantean que lesiones polipoideas pequeñas y numerosas en la ecotomografía se correlacionan con pólipos benignos $^{8,16}$, que no tienden a malignizarse en el tiempo ${ }^{17,18}$.

El tratamiento del pólipo vesicular diagnosticado por imágenes puede tomar dos rumbos: el quirúrgico, o el seguimiento ecográfico. Al respecto, Moriguchi et $\mathrm{al}^{12}$, en un estudio de seguimiento a 109 pacientes con pólipos vesiculares, en su mayoría múltiples y menores de $5 \mathrm{~mm}$, refieren que $88,3 \%$ de ellos no cambiaron de tamaño o se redujeron en un seguimiento ecográfico de 5 años. Otro estudio reciente, con seguimiento ecográfico de 12 años, mostró que 50\% de los pólipos vesiculares se mantienen del mismo tamaño, y que $23,5 \%$ disminuyó de tamaño o desapareció $^{17}$.

Sin embargo, esta conducta requiere responder preguntas acerca de cuáles son los factores de riesgo de malignización de las lesiones polipoideas diagnosticadas, entre los cuales se describe la edad, tamaño mayor a $10 \mathrm{~mm}$, sésiles, rápido 
crecimiento ecográfico, y colelitiasis asociada, que es un reconocido factor de riesgo de carcinoma vesicular ${ }^{6,8,12,15}$.

La serie en estudio muestra un mayor promedio de edad para los pacientes con lesiones adenomatosas (50 años vs 43 años), diferencia que no alcanza a ser significativa, probablemente por el escaso número de pólipos adenomatosos encontrados. La edad mayor de 50 años se considera como un factor independiente para predecir un pólipo tumoral ${ }^{16}$, y constituiría un factor de riesgo de malignidad ${ }^{3,6,8}$. Esto además se relaciona con la aparición del cáncer de vesícula que, en Chile, se produce en promedio a los 62 años ${ }^{15}$.

Respecto del género, no habría relación clara con la prevalencia de pólipos ${ }^{1,2,8}$ ni el riesgo de malignización, si bien algunas series apoyan esto último ${ }^{10,16}$. Nuestro estudio mostró una mayor frecuencia de estas lesiones en mujeres, al igual que otras series ${ }^{10,19}$, pero no se demostró su relación con el tipo de pólipo.

El tipo histológico más frecuentemente encontrado fue el pólipo de colesterol, al igual que en

\section{REFERENCIAS}

1. Koga A, Watanabe K, Fukuyama T. Diagnosis and Operative Indications for Polypoid Lesions of the Gallbladder. Arch Surg 1988; 123: 26-9.

2. Jorgensen T, Jensen H. Polyps in the Gallbladder. A prevalence study. Scand J Gastroenterol 1990; 25: 281-6.

3. Mainprize K, Gould S, Gilbert J. Surgical management of polypoid lesions of the gallbladder. $\mathrm{Br} \mathrm{J}$ Surg 2000; 87: 414-7.

4. Boulton R, Adams D. Gallbladder polyps: when to wait and when to act. Lancet 1997; 349: 817.

5. Christensen AH, Ishak KG. Benign tumors and pseudotumors of the gallbladder. Report of 180 cases. Arch Pathol 1970; 90: 423-32.

6. Terzi C, Sökmen S, SeçKin S, Albayrak L, Ugurlu M. Polypoid lesions of the gallbladder: Report of 100 cases with special reference to operative indications. Surgery 2000; 127: 622-7.

7. Kubota $K$, Bandai $Y$, Noie T, Ishizaki $Y$, Teruya $M$, MAKUUCHI M. How should polypoid lesions of the otras series nacionales e internacionales ${ }^{10,13,16,18}$. El adenoma vesicular, por su parte, es un diagnóstico poco frecuente, con prevalencias descritas en colecistectomías de 0,09\%-1,1\%10,14,16. Se ha descrito que tiene un potencial de malignización ${ }^{13}$, que aparentemente estaría asociado a su tama$\tilde{n}^{12}$. Kozuka, en una serie de 18 adenomas vesiculares, reportó presencia de cáncer en 7 de ellos, cuyo tamaño promedio fue mayor que los adenomas benignos, y restos de adenoma en 19\% de carcinomas infiltrantes ${ }^{14}$, lo que, según el autor, apoyaría la teoría de la secuencia adenomacáncer. En esta serie, la única lesión maligna se encontró en un adenoma. Sin embargo, por el escaso número de casos, y al no compararlo con el total de cánceres vesiculares, no es posible confirmar esta hipótesis.

En resumen, el tamaño sería la variable más importante a considerar en el manejo de los pólipos vesiculares, ya que se relaciona tanto con el diagnóstico de adenoma como con el riesgo de malignidad. Dado que puede ser estimado a través de la ecotomografía abdominal, consideramos este examen un buen elemento de evaluación.

gallbladder be treated in the era of laparoscopic cholecystectomy? Surgery 1995; 117: 481-7.

8. Yang H, Sun Y, Wang Z. Polypoid lesions of the gallbladder: diagnosis and indications for surgery. Br J Surg 1992; 79: 227-9.

9. Jirón Mi, Silva H, Whittle C, Fuster F, Madrid AM, Brahm J et al. Pólipos vesiculares. Segundo Taller de Consenso de la Asociación Chilena de Hepatología. Rev Méd Chile 1994; 122: 1316-7.

10. Csendes A, Becerra M, Smok G, Medina E, Maluenda F, Morales E. Prevalencia del cáncer de la vesícula biliar en colecistectomías. Rev Méd Chile 1991; 119: 887-90.

11. Moriguchi H, Tazawa J, Hayashi Y, Takenawa $H$, Nakayama E, Marumo F et al. Natural history of polypoid lesions in the gallbladder. Gut 1996; 39: 860-2.

12. Roa I, De Aretxabala X, Morgan R, Molna R, Araya JC Roa J et al. Pólipos y adenomas de la vesícula biliar: consideraciones clínico-patológicas. Rev Méd Chile 2004; 132: 673-9. 
13. Kozuka S, Tsubone M, Yasui A, Hachisuka K. Relation of Adenoma to Carcinoma in the Gallbladder. Cancer 1982; 50: 2226-34.

14. SMok G, BentJerodt R, CSENDES A. Lesiones polipoideas benignas de la vesícula biliar. Relación con adenocarcinoma vesicular. Rev Méd Chile 1992; 120: 31-5.

15. YeH CN, Jan Y, Chao TC, Chen MF. Laparoscopic cholecystectomy for polypoid lesions of the gallbladder: a clinicopathologic study. Surg Laparosc Endosc Percutan Tech 2001; 11: 176-81.

16. Окамото M, Окамото $\mathrm{H}$, Kitahara F, Ковayashi $\mathrm{K}$, Karikome K, MiURa K ET aL. Ultrasonographic
Evidence of Association of Polyps and Stones with Gallbladder Cancer. Am J Gastroenterol 1999; 94: 446-50.

17. Csendes A, Burgos AM, Csendes P, Smok G, Rojas R. Late Follow-Up of Polypoid Lesions of the Gallbladder Smaller than $10 \mathrm{~mm}$. Ann Surg 2001; 234: 657-60.

18. AldRige MC, Bismuth H. Gallbladder cancer: the polyp-cancer sequence. Br J Surg 1990; 77: 363-4.

19. Astete G, Lynch O, Madariaga J, Kawaguchi F, Bufadie ME, Martínez L et al. Lesiones elevadas de la vesícula biliar. Rev Chil Cir 1999; 51: 159-63. 